MATHEMATICS OF COMPUTATION

Volume 74, Number 252, Pages 1793-1801

S $0025-5718(05) 01717-5$

Article electronically published on March 18, 2005

\title{
COMPUTATION OF THE EIGENVALUES OF STURM-LIOUVILLE PROBLEMS WITH PARAMETER DEPENDENT BOUNDARY CONDITIONS USING THE REGULARIZED SAMPLING METHOD
}

\author{
BILAL CHANANE
}

\begin{abstract}
The purpose in this paper is to compute the eigenvalues of SturmLiouville problems with quite general separated boundary conditions nonlinear in the eigenvalue parameter using the regularized sampling method, an improvement on the method based on Shannon sampling theory, which does not involve any multiple integration and provides higher order estimates of the eigenvalues at a very low cost. A few examples shall be presented to illustrate the power of the method and a comparison made with the the exact eigenvalues obtained as squares of the zeros of the exact characteristic functions.
\end{abstract}

\section{INTRODUCTION}

In 1996, we introduced a method for computing the eigenvalues of regular SturmLiouville (SL) problems with Dirichlet boundary conditions [4]. The simple observation that the boundary (characteristic) function associated with the SL problem happens to be in a Paley-Wiener space led to the conclusion that it can be recovered from its samples at a countable number of points, using the well known WhitakerShannon-Kotel'nikov theorem. We generalized this idea to some classes of singular problems [5, 6]. Subsequently, we extended the scope of the method to include regular SL problems with general separated boundary conditions [7], SL problems with coupled self-adjoint boundary conditions [10, random SL problems [11 and regular fourth order SL problems 12. In fact, we obtained much higher order estimates at the expense of having to subtract terms involving multiple integrals [8].

The purpose of this paper is twofold. First we shall consider a regularization avoiding any (multiple) integration and show that we can get higher order estimates of the eigenvalues at a very low cost. This will constitute a substantial improvement on the original method, and we will take this opportunity to call this method the regularized sampling method. Second, we tackle the computation of the eigenvalues of Sturm-Liouville problems with quite general separated parameter dependent boundary conditions although the known theory is for boundary conditions which are affine in the parameter [1], 2], 3], 13], [18.

Received by the editor June 23, 2003 and, in revised form, March 18, 2004.

2000 Mathematics Subject Classification. Primary 34B24, 34L15, 34B07.

Key words and phrases. Second order Sturm-Liouville problems, eigenvalue problems, Whittaker-Shannon-Kotel'nikov theorem, parameter dependent boundary conditions, regularized sampling method. 


\section{MAIN RESUlts}

We are interested in finding the eigenvalues of the following SL problem with separated parameter dependent boundary conditions:

$$
\left\{\begin{array}{c}
-y^{\prime \prime}+q y=\mu^{2} y, \quad x \in[0, \gamma], \\
a_{11}(\mu) y(0)-a_{12}(\mu) y^{\prime}(0)=0, \\
a_{21}(\mu) y(\gamma)+a_{22}(\mu) y^{\prime}(\gamma)=0,
\end{array}\right.
$$

where $a_{i j}$ are entire functions satisfying the growth conditions

$$
\left|a_{i j}(\mu)\right| \leq c_{i j}(1+|\mu|)^{m_{0}} \exp \left(L_{i j}|\operatorname{Im} \mu|\right), 1 \leq i, j \leq 2,
$$

$a_{11}^{2}(\mu)+a_{12}^{2}(\mu) \neq 0, a_{22}^{2}(\mu)+a_{21}^{2}(\mu) \neq 0, m_{0}$ is a nonnegative integer, and $q \in$ $L^{1}([0, \gamma])$. Let $c_{0}=\max c_{i j}, L=\max L_{i j}$.

We shall assume further that the definiteness conditions

$$
\left\{\begin{array}{l}
a_{11} \mu \frac{d a_{12}}{d \mu}-a_{12} \mu \frac{d a_{11}}{d \mu} \geq 0 \\
a_{21} \mu \frac{d a_{22}}{d \mu}-a_{22} \mu \frac{d a_{21}}{d \mu} \leq 0
\end{array}\right.
$$

or

$$
\left\{\begin{array}{c}
a_{11} \mu \frac{d a_{12}}{d \mu}-a_{12} \mu \frac{d a_{11}}{d \mu} \leq 0, \\
a_{21} \mu \frac{d a_{22}}{d \mu}-a_{22} \mu \frac{d a_{21}}{d \mu} \geq 0
\end{array}\right.
$$

be satisfied (at least one inequality must be strict in any one of these conditions to make the miss distance function strictly monotone [16]). This is to ensure a monotone Prufer miss distance which guarantees at most one eigenvalue $\lambda_{k}=\mu_{k}^{2}$ of any index $k$ having an eigenfunction with just $k$ zeros in $(0, \gamma)$.

Consider the two base problems

$$
\left\{\begin{array}{c}
-y^{\prime \prime}+q y=\mu^{2} y, \quad x \in[0, \gamma] \\
y(0, \mu)=1, y^{\prime}(0, \mu)=0
\end{array}\right.
$$

and

$$
\left\{\begin{array}{c}
-y^{\prime \prime}+q y=\mu^{2} y, \quad x \in[0, \gamma], \\
y(0, \mu)=0, y^{\prime}(0, \mu)=1,
\end{array}\right.
$$

whose solutions are denoted $y_{1}$ and $y_{2}$, respectively. The solution to the initial value problem

$$
\left\{\begin{array}{c}
-y^{\prime \prime}+q y=\mu^{2} y, \quad x \in[0, \gamma] \\
y(0, \mu)=a_{12}(\mu), y^{\prime}(0, \mu)=a_{11}(\mu)
\end{array}\right.
$$

and its derivative are, therefore,

$$
\left\{\begin{array}{c}
y(x, \mu)=a_{12}(\mu) y_{1}(x, \mu)+a_{11}(\mu) y_{2}(x, \mu), \\
y^{\prime}(x, \mu)=a_{12}(\mu) y_{1}^{\prime}(x, \mu)+a_{11}(\mu) y_{2}^{\prime}(x, \mu) .
\end{array}\right.
$$

It is well known that $y_{1}(x, \mu)-\cos \mu x, y_{1}^{\prime}(x, \mu)+\mu \sin \mu x, y_{2}(x, \mu)$ and $y_{2}^{\prime}(x, \mu)-$ $\cos \mu x$ are entire functions of $\mu$ for each $x \in(0, \gamma]$ and satisfy the same growth condition

$$
\begin{gathered}
\left|y_{1}(x, \mu)-\cos \mu x\right|, \quad\left|y_{1}^{\prime}(x, \mu)+\mu \sin \mu x\right|, \quad\left|y_{2}(x, \mu)\right|, \\
\left|y_{2}^{\prime}(x, \mu)-\cos \mu x\right| \leq c_{1} \exp (x|\operatorname{Im} \mu|)
\end{gathered}
$$


for some constant $c_{1}$. In $\left[\underline{8}\right.$, we have shown that $y_{1}(x, \mu)-\cos \mu x, y_{1}^{\prime}(x, \mu)+$ $\mu \sin \mu x-\int_{0}^{x} q(t) \cos \mu(x-t) \cos \mu t d t, y_{2}(x, \mu)-\frac{\sin \mu x}{\mu x}$ and $y_{2}^{\prime}(x, \mu)-\cos \mu x-$ $\int_{0}^{x} q(t) \cos \mu(x-t) \frac{\sin \mu t}{\mu t} d t$ are entire functions of $\mu$ for each $x \in(0, \gamma]$ and satisfy the same growth condition

$$
\begin{aligned}
& \left|y_{1}(x, \mu)-\cos \mu x\right|,\left|y_{1}^{\prime}(x, \mu)+\mu \sin \mu x-\int_{0}^{x} q(t) \cos \mu(x-t) \cos \mu t d t\right|, \\
& \left|y_{2}(x, \mu)-\frac{\sin \mu x}{\mu x}\right|,\left|y_{2}^{\prime}(x, \mu)-\cos \mu x-\int_{0}^{x} q(t) \cos \mu(x-t) \frac{\sin \mu t}{\mu t} d t\right| \\
\leq & \frac{c_{2}}{1+\gamma|\mu|} \exp (x|\operatorname{Im} \mu|),
\end{aligned}
$$

which means that they belong to the Paley-Wiener space $P W_{x}$ defined by

$$
P W_{x}=\left\{h(z) \text { entire } /|h(z)| \leq C \exp \{x|z|\}, \int_{-\infty}^{\infty}|h(z)|^{2} d z<\infty\right\} .
$$

In fact we have obtained much higher order estimates at the expense of having to subtract from $y_{i}$ and $y_{i}^{\prime}$ terms involving multiple integrals. In this paper, we shall stick with the first estimate given, hence avoiding any (multiple) integration and show by the same token that we can get a higher order estimate at a very low cost. Let

$$
B(x, \mu)=a_{21}(\mu) y(x, \mu)+a_{22}(\mu) y^{\prime}(x, \mu) .
$$

The eigenvalues of the problem are seen as the square of the zeros of the boundary (characteristic) function $B(\gamma, \mu)$.

Replacing $y(x, \mu)$ and $y^{\prime}(x, \mu)$ by their expressions, we obtain

$$
\begin{aligned}
B(x, \mu)= & a_{21}(\mu)\left\{a_{12}(\mu) y_{1}(x, \mu)+a_{11}(\mu) y_{2}(x, \mu)\right\} \\
& +a_{22}(\mu)\left\{a_{12}(\mu) y_{1}^{\prime}(x, \mu)+a_{11}(\mu) y_{2}^{\prime}(x, \mu)\right\}
\end{aligned}
$$

from which we get

$$
B(x, \mu)=B_{0}(x, \mu)+B_{1}(x, \mu),
$$

where

$$
\left\{\begin{array}{c}
B_{0}(x, \mu)=a_{21}(\mu)\left\{a_{12}(\mu)\left[y_{1}(x, \mu)-\cos \mu x\right]+a_{11}(\mu) y_{2}(x, \mu)\right\} \\
+a_{22}(\mu)\left\{a_{12}(\mu)\left[y_{1}^{\prime}(x, \mu)+\mu \sin \mu x\right]+a_{11}(\mu)\left[y_{2}^{\prime}(x, \mu)-\cos \mu x\right]\right\}, \\
B_{1}(x, \mu)=a_{21}(\mu) a_{12}(\mu) \cos \mu x+a_{22}(\mu) a_{12}(\mu) \mu \sin \mu x+a_{22}(\mu) a_{11}(\mu) \cos \mu x .
\end{array}\right.
$$

Theorem 2.1. $B_{0}$ is an entire function of $\mu$ for each $x \in(0, \gamma]$ and satisfies the growth condition

$$
\left|B_{0}(x, \mu)\right| \leq c_{2}(1+|\mu|)^{2 m_{0}} \exp ((2 L+x)|\operatorname{Im} \mu|) .
$$

Proof. $B_{0}$ is an entire function of $\mu$ for each $x \in(0, \gamma]$ as a sum of products of entire functions. As for the estimate, we have

$$
\begin{aligned}
\left|B_{0}(x, \mu)\right| \leq & \left|a_{21}(\mu)\right|\left\{\left|a_{12}(\mu)\right|\left|y_{1}(x, \mu)-\cos \mu x\right|+\left|a_{11}(\mu)\right| y_{2}(x, \mu)\right\} \\
& +\left|a_{22}(\mu)\right|\left\{\left|a_{12}(\mu)\right|\left|y_{1}^{\prime}(x, \mu)+\mu \sin \mu x\right|+\left|a_{11}(\mu)\right|\left|y_{2}^{\prime}(x, \mu)-\cos \mu x\right|\right\} \\
\leq & c_{2}(1+|\mu|)^{2 m_{0}} \exp ((2 L+x)|\operatorname{Im} \mu|)
\end{aligned}
$$

with $c_{2}=4 c_{0}^{2} c_{1}$. 
Corollary 2.2. $\left(\frac{\sin \theta \mu}{\theta \mu}\right)^{m} B_{0}(\gamma, \mu)$ is an entire function of $\mu$ and satisfies the estimate

$$
\left|\left(\frac{\sin \theta \mu}{\theta \mu}\right)^{m} B_{0}(\gamma, \mu)\right| \leq \frac{c_{3}}{(1+\theta|\mu|)^{m-2 m_{0}}} \exp ((2 L+\gamma+m \theta)|\operatorname{Im} \mu|)
$$

for a positive integer $m \geq 2 m_{0}+2$ and positive constants $c_{3}$ and $\vartheta$.

Proof. It is enough to note that $\frac{\sin \theta \mu}{\theta \mu}$ is an entire function of $\mu$ and use the standard estimate $\left|\frac{\sin z}{z}\right| \leq \frac{c_{4}}{1+|z|} \exp (|\operatorname{Im} z|)$, where $c_{4}=1.72$ (say), $c_{3}=c_{2} c_{4}^{m}$, and the above theorem.

Thus, $h(\mu)=\left(\frac{\sin \theta \mu}{\theta \mu}\right)^{m} B_{0}(\gamma, \mu)$ belongs to the Paley-Wiener space $P W_{\sigma}$ with $\sigma=2 L+\gamma+m \theta$. Hence, $h$ can be recovered from its values at the points $\mu_{j}=j \frac{\pi}{\sigma}$, $j \in \mathbb{Z}$, using the following celebrated theorem.

Theorem 2.3 (Whitaker-Shannon-Kotel'nikov). Let $h \in P W_{\sigma}$, then

$$
h(\mu)=\sum_{j=-\infty}^{\infty} h\left(\mu_{j}\right) \frac{\sin \sigma\left(\mu-\mu_{j}\right)}{\sigma\left(\mu-\mu_{j}\right)}
$$

$\mu_{j}=j \frac{\pi}{\sigma}$. The series converges absolutely and uniformly on compact subsets of $\mathbb{C}$ and in $L_{d \mu}^{2}(\mathbb{R})$.

Now, since $\mu^{m-2 m_{0}-1} h(\mu) \in L^{2}(-\infty, \infty)$, Jagerman's result (see [19, Theorem 3.21, p.90) is applicable and yields the following very sharp estimate.

Lemma 2.4 (Truncation error). Let $h_{N}(\mu)=\sum_{j=-N}^{N} h\left(\mu_{j}\right) \frac{\sin \sigma\left(\mu-\mu_{j}\right)}{\sigma\left(\mu-\mu_{j}\right)}$ denote the truncation of $h(\mu)$. Then, for $|\mu|<N \pi / \sigma$,

$$
\begin{aligned}
& \left|h(\mu)-h_{N}(\mu)\right| \\
& \quad \leq \frac{|\sin \gamma \mu| c_{5}}{\pi(\pi / \sigma)^{m-2 m_{0}-1} \sqrt{1-4^{-m+2 m_{0}+1}}}\left[\frac{1}{\sqrt{(N \pi / \sigma)-\mu}}+\frac{1}{\sqrt{(N \pi / \sigma)+\mu}}\right] \frac{1}{(N+1)^{m-2 m_{0}-1}}, \\
& \text { where } c_{5}=\|\left.\mu^{m-2 m_{0}-1} h(\mu)\right|_{2} .
\end{aligned}
$$

Let $\bar{\mu}^{2}$ be an exact eigenvalue and $\mu_{N}^{2}$ the corresponding approximation obtained as a square of a zero of $\left[\left(\frac{\sin \theta \mu}{\theta \mu}\right)^{-m+2 m_{0}} h_{N}(\mu)+B_{1}(\gamma, \mu)\right]$. Then from

$$
\begin{aligned}
& \left|B(\gamma, \mu)-\left[\left(\frac{\sin \theta \mu}{\theta \mu}\right)^{-m+2 m_{0}} h_{N}(\mu)+B_{1}(\gamma, \mu)\right]\right| \\
& =\left|\left(\frac{\sin \theta \mu}{\theta \mu}\right)^{-m+2 m_{0}}\left[h(\mu)-h_{N}(\mu)\right]\right| \\
& \leq\left|\frac{\sin \theta \mu}{\theta \mu}\right|^{-m+2 m_{0}} \frac{|\sin \gamma \mu| c_{5}}{\pi(\pi / \sigma)^{m-2 m_{5} 1} \sqrt{1-4^{-m+2 m_{0}+1}}} \\
& \times\left[\frac{1}{\sqrt{(N \pi / \sigma)-\mu}}+\frac{1}{\sqrt{(N \pi / \sigma)+\mu}}\right] \frac{1}{(N+1)^{m-2 m_{0}-1}}
\end{aligned}
$$


we get

$$
\begin{aligned}
\left|B\left(\gamma, \mu_{N}\right)-B(\gamma, \bar{\mu})\right| & \left|B\left(\gamma, \mu_{N}\right)-\left[\left(\frac{\sin \theta \mu_{N}}{\theta \mu_{N}}\right)^{-m+2 m_{0}} h_{N}\left(\mu_{N}\right)+B_{1}\left(\gamma, \mu_{N}\right)\right]\right| \\
\leq & \left|\frac{\sin \theta \mu_{N}}{\theta \mu_{N}}\right|^{-m+2 m_{0}} \frac{\left|\sin \gamma \mu_{N}\right| c_{5}}{\pi(\pi / \sigma)^{m-2 m_{0}-1 \sqrt{1-4-m+2 m_{0}+1}}} \\
& \times\left[\frac{1}{\sqrt{(N \pi / \sigma)-\mu_{N}}}+\frac{1}{\sqrt{(N \pi / \sigma)+\mu_{N}}}\right] \frac{1}{(N+1)^{m-2 m_{0}-1}},
\end{aligned}
$$

but $\left|B\left(\gamma, \mu_{N}\right)-B(\gamma, \bar{\mu})\right|=\left|B_{\mu}(\gamma, \xi)\right|\left|\mu_{N}-\bar{\mu}\right|$ for some $\xi$ in a small ball centered at $\mu_{N}$ with radius $\left|\mu_{N}-\bar{\mu}\right|$ and not containing a multiple of $\pi / \theta$. Hence,

Theorem 2.5 (Error bounds). For $\left|\mu_{N}\right|<N \pi / \sigma$,

$$
\begin{aligned}
\left|\mu_{N}-\bar{\mu}\right| \leq & \frac{1}{\inf \left|B_{\mu}(\gamma, \xi)\right|}\left|\frac{\sin \theta \mu_{N}}{\theta \mu_{N}}\right|^{-m+2 m_{0}} \frac{\left|\sin \gamma \mu_{N}\right| c_{5}}{\pi(\pi / \sigma)^{m-2 m_{0}-1} \sqrt{1-4^{-m+2 m_{0}+1}}} \\
& \times\left[\frac{1}{\sqrt{(N \pi / \sigma)-\mu_{N}}}+\frac{1}{\sqrt{(N \pi / \sigma)+\mu_{N}}}\right] \frac{1}{(N+1)^{m-2 m_{0}-1}},
\end{aligned}
$$

where the inf is taken over a ball centered at $\mu_{N}$ with radius $\left|\mu_{N}-\bar{\mu}\right|$ and does not contain a multiple of $\pi / \theta$.

\section{Numerical EXAmples}

In this section, we shall present a few examples to illustrate our method. We have taken $\theta=(2 L+\gamma) /(N-m)$ in order to avoid the first singularity of $\left(\frac{\sin \theta \mu_{N}}{\theta \mu_{N}}\right)^{-1}$. The sampling values were obtained using the Fehlberg 4-5 order Runge-Kutta method.

Example 3.1 (Taken from Binding and Browne [2]).

$$
\left\{\begin{array}{c}
-y^{\prime \prime}(x)=\lambda y(x), 0 \leq x \leq 1, \\
-y(0)=(\lambda+d) y^{\prime}(0), \\
y(1)=\lambda y^{\prime}(1)
\end{array}\right.
$$

where $d=-4 \pi^{2}$. We took $L=1, m=2, N=40$, and a precision of $10^{-10}$. The computed eigenvalues together with the "exact" ones are displayed in Table 3.1 . As pointed out in [2], the oscillation counts occur in the sequence $1,3,4,5, \ldots$, the minimal count is 1 not 0 , and there is count 2 missing. The exact characteristic function is

$$
B_{\text {exact }}(\mu)=\left(1+4 \pi^{2} \mu^{4}-\mu^{6}\right) \frac{\sin \mu}{\mu}-\left(2 \mu^{2}-4 \pi^{2}\right) \cos \mu,
$$

where zero is not an eigenvalue.

TABLE 3.1. The first three eigenvalues in Example 3.1

\begin{tabular}{|c|c|c|c|}
\hline Index & Exact Eigenvalues & Approximate Eigenvalues & Absolute Error \\
\hline 1 & 9.730886578213082033 & 9.730887696302056807 & 0.00000111808897477 \\
\hline 2 & 88.76331625258976337 & 88.76323738197181406 & 0.00007887061794931 \\
\hline 3 & 157.88411043863472059 & 157.88422274978466468 & 0.00011231114994409 \\
\hline
\end{tabular}


TABLE 3.2a. The first three eigenvalues in Example 3.2

\begin{tabular}{|c|c|c|c|}
\hline Index & Exact Eigenvalues & Approximate Eigenvalues & Absolute Error \\
\hline 1 & 9.929679054283188 & 0.929678898812778 & $0.155470411 \times 10^{-6}$ \\
\hline 2 & 9.9387434140 & 9.9387439016 & $4.8758708700 \times 10^{-7}$ \\
\hline 3 & 11.2738742105212 & 11.2738738490945 & $-0.3614267 \times 10^{-6}$ \\
\hline
\end{tabular}

TABLE 3.2b. Eigenvalues from different ranges for Example 3.2

\begin{tabular}{|c|c|c|c|}
\hline Exact Eigenvalues & Approximate Eigenvalues & Relative Error & Absolute Error \\
\hline $1.0169749051 \times 10^{6}$ & $1.0169749052 \times 10^{6}$ & $-3.882156763 \times 10^{-11}$ & 0.000136868 \\
\hline $1.00119488705 \times 10^{8}$ & $1.00119488709 \times 10^{8}$ & $3.882156763 \times 10^{-11}$ & 0.00388679 \\
\hline $1.000126382927378 \times 10^{10}$ & $1.00012638292493 \times 10^{10}$ & $2.44062879930 \times 10^{-12}$ & 0.024409 \\
\hline
\end{tabular}

\section{Example 3.2.}

$$
\left\{\begin{array}{c}
-y^{\prime \prime}(x)=\lambda y(x), 0 \leq x \leq 1, \\
y(0)-2 y^{\prime}(0)=0, \\
(1+\sqrt{\lambda}) y(1)+(1-\lambda) y^{\prime}(1)=0 .
\end{array}\right.
$$

We took $L=0, m_{0}=2, N=40$, and a precision of $10^{-10}$. The computed eigenvalues $\lambda=\mu^{2}$ together with the "exact" ones are displayed in Table 3.2a, The exact characteristic function is

$$
B_{\text {exact }}(\mu)=(2 \cos (\mu)+\sin (\mu) / \mu)+(1-\mu)(-2 \mu \sin (\mu)+\cos (\mu)) .
$$

1 is not an eigenvalue.

It is appropriate to note how perfect the approximation is over different ranges by checking Table $3.2 \mathrm{~b}$, in which we include both the absolute and relative error.

Example 3.3 (Taken from Pryce [16], an "indefinite" case).

$$
\left\{\begin{array}{c}
-y^{\prime \prime}(x)=\lambda y(x), 0 \leq x \leq \pi / 2, \\
y^{\prime}(0)=\lambda\left(\frac{3}{2} y(0)+y^{\prime}(0)\right), \\
y^{\prime}(\pi / 2)=0 .
\end{array}\right.
$$

This example illustrates the case in which the definiteness condition is not satisfied. We have three eigenvalues $\lambda_{1}=0, \lambda_{2}=1 / 4, \lambda_{3}=1$ to which correspond three eigenfunctions $y_{1}(x)=1, y_{2}(x)=\sin \frac{x}{2}+\cos \frac{x}{2}, y_{3}(x)=\sin x$. We took $L=1$, $m=2, N=30$, and a precision of $10^{-10}$. The computed eigenvalues $\lambda=\mu^{2}$ together with the "exact" ones are displayed in Table 3.3. The exact characteristic function is

$$
B_{\text {exact }}(\mu)=-\left(1-\mu^{2}\right) \mu \sin \left(\mu \frac{\pi}{2}\right)+\frac{3}{2} \mu^{2} \cos \left(\mu \frac{\pi}{2}\right) .
$$

TABLE 3.3. The first three eigenvalues in Example 3.3

\begin{tabular}{|c|c|c|c|}
\hline Index & Exact Eigenvalues & Approximate Eigenvalues & Absolute Error \\
\hline 1 & 0 & $1.2857880801746297 \times 10^{-10}$ & $1.2857880801746297 \times 10^{-10}$ \\
\hline 2 & $1 / 4$ & 0.24999814884168953010 & $1.8511583104698968 \times 10^{-6}$ \\
\hline 3 & 1 & 0.9999881573635478785 & $1.18426364521215 \times 10^{-5}$ \\
\hline
\end{tabular}




\section{Example 3.4.}

$$
\left\{\begin{array}{c}
-y^{\prime \prime}(x)+e^{x} y(x)=\lambda y(x), 0 \leq x \leq 1, \\
y(0)=0, \\
-\sqrt{\lambda} \sin (\sqrt{\lambda}) y(1)+\cos (\sqrt{\lambda}) y^{\prime}(1)=0 .
\end{array}\right.
$$

It is easy to check that the definiteness condition is satisfied. We took $L=1$, $m_{0}=1, N=40$, and a precision of $10^{-10}$. The computed eigenvalues $\lambda=\mu^{2}$ together with the "exact" ones are displayed in Table 3.4. Here again we are in a position to derive the exact characteristic function which in fact can be expressed in terms of the modified Bessel functions of the first kind. Indeed, let $\lambda=\mu^{2}$ and consider the change of variables $t=2 \mathbf{e}^{x / 2}$ and $\nu=2 \iota \mu$, where $\iota=\sqrt{-1}$. The differential equation becomes the modified Bessel equation of order $\nu$ given by

$$
t^{2} \frac{d^{2} z}{d t^{2}}+t \frac{d z}{d t}-\left(t^{2}+\nu^{2}\right) z=0
$$

whose solution is

$$
z(t)=c_{1} \mathcal{I}_{\nu}(t)+c_{2} \mathcal{I}_{-\nu}(t),
$$

where $\mathcal{I}_{\nu}$ and $\mathcal{I}_{-\nu}$ are the modified Bessel functions of the first kind of order $\nu$.

Returning to the original variables, we obtain

$$
y(x)=c_{1} \mathcal{I}_{2 \iota \mu}\left(2 \mathbf{e}^{x / 2}\right)+c_{2} \mathcal{I}_{-2 \iota \mu}\left(2 \mathbf{e}^{x / 2}\right) .
$$

Taking into account the boundary conditions, we obtain the homogeneous system in $c_{1}$ and $c_{2}$

$$
\left\{\begin{array}{c}
c_{1} \mathcal{I}_{2 \iota \mu}(2)+c_{2} \mathcal{I}_{-2 \iota \mu}(2)=0 \\
c_{1}\left\{-\mu \mathcal{I}_{2 \iota \mu}(2 \sqrt{\mathbf{e}}) \sin \mu+\sqrt{\mathbf{e}} \mathcal{I}_{2 \iota \mu}^{\prime}(2 \sqrt{\mathbf{e}}) \cos \mu\right\} \\
+c_{2}\left\{-\mu \mathcal{I}_{-2 \iota \mu}(2 \sqrt{\mathbf{e}}) \sin \mu+\sqrt{\mathbf{e}} \mathcal{I}_{-2 \iota \mu}^{\prime}(2 \sqrt{\mathbf{e}}) \cos \mu\right\}=0
\end{array}\right.
$$

In order to have a nontrivial solution, a necessary and sufficient condition is to have $B_{\text {exact }}(\mu)=0$ where

$$
\begin{aligned}
B_{\text {exact }}(\mu) & \mathcal{I}_{-2 \iota \mu}(2) \\
=\iota \mid & \mathcal{I}_{2 \iota \mu}(2) \\
-\mu \mathcal{I}_{2 \iota \mu}(2 \sqrt{\mathbf{e}}) \sin \mu+\sqrt{\mathbf{e}} \mathcal{I}_{2 \iota \mu}^{\prime}(2 \sqrt{\mathbf{e}}) \cos \mu & -\mu \mathcal{I}_{-2 \iota \mu}(2 \sqrt{\mathbf{e}}) \sin \mu+\sqrt{\mathbf{e}} \mathcal{I}_{-2 \iota \mu}^{\prime}(2 \sqrt{\mathbf{e}}) \cos \mu
\end{aligned} \mid
$$

is the characteristic function. The $\iota$ in front of the determinant makes $B_{\text {exact }}$ a real function. Now, using the well known result

$$
\frac{d}{d x}\left[x^{\nu} \mathcal{I}_{\nu}(x)\right]=x^{\nu} \mathcal{I}_{\nu-1}(x),
$$

TABLE 3.4. The first six eigenvalues in Example 3.4

\begin{tabular}{|c|c|c|c|}
\hline Index & Exact Eigenvalues & Approximate Eigenvalues & Absolute Error \\
\hline 1 & 0.92906202857 & 0.9290620353 & $6.75215627149 \times 10^{-9}$ \\
\hline 2 & 6.7478811782 & 6.7478811410 & $3.7173601254 \times 10^{-8}$ \\
\hline 3 & 16.1245477258 & 16.1245478044 & $7.8559790579 \times 10^{-8}$ \\
\hline 4 & 31.2202765051 & 31.22027698028 & $4.7517563634 \times 10^{-7}$ \\
\hline 5 & 50.73392783919 & 50.73392843916 & $5.9996614290 \times 10^{-7}$ \\
\hline 6 & 75.5814691882 & 75.5814692597 & $7.1549766566 \times 10^{-8}$ \\
\hline
\end{tabular}


we obtain

$$
\begin{aligned}
& B_{\text {exact }}(\mu) \\
& =\mathcal{I}_{2 \iota \mu}(2)\left\{-\mu \mathcal{I}_{-2 \iota \mu}(2 \sqrt{\mathbf{e}}) \sin \mu+\sqrt{\mathbf{e}}\left[\mathcal{I}_{-2 \iota \mu-1}(2 \sqrt{\mathbf{e}})+\frac{\iota \mu}{\sqrt{\mathbf{e}}} \mathcal{I}_{-2 \iota \mu}(2 \sqrt{\mathbf{e}})\right] \cos \mu\right\} \\
& \quad-\iota \mathcal{I}_{-2 \iota \mu}(2)\left\{-\mu \mathcal{I}_{2 \iota \mu}(2 \sqrt{\mathbf{e}}) \sin \mu+\sqrt{\mathbf{e}}\left[\mathcal{I}_{2 \iota \mu-1}(2 \sqrt{\mathbf{e}})-\frac{\iota \mu}{\sqrt{\mathbf{e}}} \mathcal{I}_{2 \iota \mu}(2 \sqrt{\mathbf{e}})\right] \cos \mu\right\} .
\end{aligned}
$$

\section{Conclusion}

In this paper, we have improved upon the method based on Shannon sampling theory introduced in [4 by considering a regularization avoiding any multiple integration and shown that we can get higher order estimates of the eigenvalues at a very low cost. We shall call this method the regularized sampling method. We have presented a few examples to illustrate a method and compared the computed eigenvalues with the exact ones obtained as squares of the zeros of the exact characteristic functions.

\section{ACKNOWLEDGMENTS}

This work was completed while the author was visiting Cardiff University, UK. The author wishes to thank Professor W. D. Evans and the Mathematics Department for their hospitality and stimulating atmosphere during his stay. The author also wishes to thank King Fahd University of Petroleum and Minerals for its constant support, and by making this possible through grant MS/SPECTRAL/269.

\section{REFERENCES}

1. P. A. Binding, P. J. Browne, K. Seddighi, Sturm-Liouville problem with eigenparameter dependent boundary conditions, Proc. Edinburgh Math. Soc. (2), 37, No. 1, (1994), 57-72. MR 95k:34039

2. P. A. Binding, P. J. Browne, Oscillation theory for indefinite Sturm-Liouville problems with eigenparameter-dependent boundary conditions, Proc. Royal Soc. Edinburgh, 127A, (1997), 1123-1136. MR98j:34037

3. A. Boumenir, The sampling method for Sturm-Liouville problems with the eigenvalue parameter in the boundary condition, Numer. Funct. Anal. Optim., 21, No. 1-2, (2000), 67-75. MR 2001c:34175

4. A. Boumenir, B. Chanane, Eigenvalues of Sturm-Liouville systems using sampling theory, Appl. Anal., 62, (1996), 323-334.

5. A. Boumenir and B. Chanane, Computing eigenvalues of Sturm-Liouville systems of Bessel type, Proc. Edinburgh Math. Soc., 42, (1999), 257-265. MR2000g:65071

6. A. Boumenir and B. Chanane, The computation of negative eigenvalues of singular SturmLiouville problems, I.M.A. J. Num. Anal., 21, (2), (2001), 489-501. MR2002a:65120

7. B. Chanane, Computing Eigenvalues of Regular Sturm-Liouville Problems, Appl. Math. Lett., Vol.12, (1999), 119-125. MR2000k:34129

8. B. Chanane, High Order Approximations of Eigenvalues of Regular Sturm-Liouville Problems, J. Math. Anal. Appl., 226, (1998), 121-129. MR99m:65144

9. B. Chanane, The Paley-Wiener-Levinson theorem and the computation of Sturm-Liouville eigenvalues: Irregular sampling, Appl. Anal., Vol.75 (3-4), (2000), 261-266. MR2001i:34144

10. B. Chanane, High Order Approximations of the Eigenvalues of Sturm-Liouville Problems with Coupled Self-Adjoint Boundary Conditions, Appl. Anal., Vol. 80, (2001), 317-330. MR 2003f:34166

11. B. Chanane, On a class of random Sturm-Liouville problems, Int. J. Appl. Math., Vol.8, No.2, (2002), 171-182. MR 2002i:34033 
12. B. Chanane, Eigenvalues of Regular Fourth Order Sturm-Liouville Problems using Interpolation Theory, Approximation Theory X: Wavelets, Splines, and Applications, Charles K. Chui, Larry L. Schumaker, and Joachim Stockler (eds.), The Vanderbilt University Press, Innovations in Applied Mathematics, (2002), 155-166. MR.2003g:65090

13. C. T. Fulton, Two point boundary value problems with eigenvalue parameter contained in the boundary conditions, Proc. Roy. Soc. Edinburgh 77, (1977), 293-308. MR58:28804

14. D. Hinton, P. W. Schaefer, Spectral Theory and Computational Methods of Sturm-Liouville Problems, Marcel Dekker, Inc. MR98b:34003

15. Naimark, M. A., Linear Differential Operators II, G.G. Harrap \& Co. Ltd, 1968. MR41:7485

16. J. D. Pryce, Numerical Solution of Sturm-Liouville Problems, Oxford Science Publications, Clarendon Press, 1993. MR 95h:65056

17. F. Stenger, Numerical Methods Based on Sinc and Analytic Functions, Springer-Verlag., 1993. MR $94 \mathrm{k}: 65003$

18. J. Walter, Regular eigenvalue problems with eigenvalue parameter in the boundary conditions, Math. Z. 133, (1973), 301-312. MR 49:713

19. A. I. Zayed, Advances in Shannon's Sampling Theory, CRC Press, Boca Raton, FL, 1993. MR $95 \mathrm{f}: 94008$

Department of Mathematical Sciences, KFupm, Dhahran 31261, Saudi Arabia

E-mail address: chanane@kfupm.edu.sa 